suggest that qualitative methods training be instituted as part of the gold standard of I-O psychology training. We hope, too, that the success stories we outlined above underscore the rigor and quality of qualitative methods in both research and applied endeavors. Moreover, we hope that qualitative methods training can be met not with begrudging acceptance but with enthusiasm, as a valuable skill set. As organizational scientists, we study vexing and complex problems. We need at our disposal all types of tools, approaches, and philosophies in our attempt to make a positive change, seek truth, and advance our science/practice.

\title{
References
}

Berka, G., Lopina, E., Justice, L., \& Beck, T. (2014). Southern Chair Company: We won't take this sitting down. Case Research Journal, 34(2), 83-105.

Lindlof, T. R., \& Taylor, B. C. (2011). Qualitative communication research methods (3rd ed.). Thousand Oaks, CA: Sage.

Long, S. D., Dunn, A. M., Makkawy, A., Uhrich, B., \& Olien, J. (2013). A culture of paradoxes: An interpretative phenomenological approach to virtual work. Electronic Journal of Communication, 23(4). Retrieved from http://www.cios.org/EJCPUBLIC/023/4/023046.html

Miles, M. B., \& Huberman, A. M. (1994). Qualitative data analysis: An expanded sourcebook (2nd ed.). Thousand Oaks, CA: Sage.

Pratt, M. G., \& Bonaccio, S. (2016). Qualitative research in I-O psychology: Maps, myths, and moving forward. Industrial and Organizational Psychology: Perspectives on Science and Practice, 9(4), 693-715.

Scott, G., \& Garner, R. (2013). Doing qualitative research: Designs, methods, and techniques. Upper Saddle River, NJ: Pearson.

Spector, P. E., Rogelberg, S. G., Ryan, A. M., Schmitt, N., \& Zedeck, S. (2014). Moving the pendulum back to the middle: Reflections on and introduction to the inductive research [Special issue]. Journal of Business and Psychology, 29, 499-502. doi:10.1007/s10869-014-9372-7

Van Maanen, J. (1988). Tales of the field: On writing ethnography. Chicago, IL: University of Chicago Press.

\section{It's Like Doing a Job Analysis: You Know More About Qualitative Methods Than You May Think}

\author{
Alice M. Brawley \\ Michigan State University \\ Cynthia L. S. Pury \\ Clemson University
}

Through learning about and doing job analysis, industrial-organizational (I-O) psychologists likely already possess skills and knowledge relevant

Alice M. Brawley, Department of Psychology, Michigan State University; Cynthia L. S. Pury, Department of Psychology, Clemson University.

Correspondence concerning this article should be addressed to Alice M. Brawley, 316 Physics Road, Room 346, Department of Psychology, Michigan State University, East Lansing, MI 48824. E-mail: brawley2@msu.edu 
to doing and understanding qualitative research. We'll illustrate this by showing similarities between common job analysis practices and one particular qualitative research approach likely to be relevant to organizational research: grounded theory. Grounded theory was "discovered" in 1967 by Barney Glaser and Anselm Strauss (Glaser \& Strauss, 1967). Though Glaser and Strauss later split in their methodologies (an occurrence not unlike the varied approaches to job analysis), the core idea of grounded theory is to develop a new theory of some process or phenomenon from the "ground" up. In the grounded theory approach, researchers typically collect mostly qualitative data - often including interviews (Creswell, 2007) — and simultaneously develop increasingly abstract codes, concepts, and categories from the data. In the final step of analysis, researchers develop a theory that subsumes all categories from the data. If researchers follow the Straussian tradition, categories can be fit into a theoretical framework that details a central phenomenon underlying the process of interest and the conditions that precede it, result from it, and shape the resulting categories (Creswell, 2007). We illustrate this framework in Figure 1. Grounded theory is particularly useful for developing an accurate understanding of many organizational processes and phenomena that I-O psychologists study.

Pratt and Bonaccio (2016) point out that there is little training offered to I-O graduate students on qualitative methods. However, some qualitative method training is undoubtedly offered to students through job analysis, and promisingly, job/task/work analysis/competency modeling and classification is listed as one of the core I-O domains that the Society for Industrial and Organizational Psychology recommends to be covered in master's level programs. As noted by the Society for Industrial and Organizational Psychology, the core concern in this domain is "obtain[ing] descriptive information" about a variety of work and worker attributes (Society for Industrial and Organizational Psychology, 2016, p. 10). In other words, job analysis involves gathering and summarizing ideas and concepts rather than only providing quantitative summaries of quantitative data. When I-O psychologists do a job analysis, we want to know what it is that workers do on the job.

Our comparison of job analysis and grounded theory comes with one very important disclaimer: Our intention is not to diminish the value of specialized training in qualitative methods. There is distinct value to be gained from in-depth study and practice of the many various qualitative research approaches, and there are important differences between job analysis and qualitative research that we discuss later in our article. Rather, our goal is to point out the knowledge and skills that all I-O psychologists are likely to have-from job analysis-that can facilitate doing and understanding qualitative research and therefore show that job analysis is a viable 


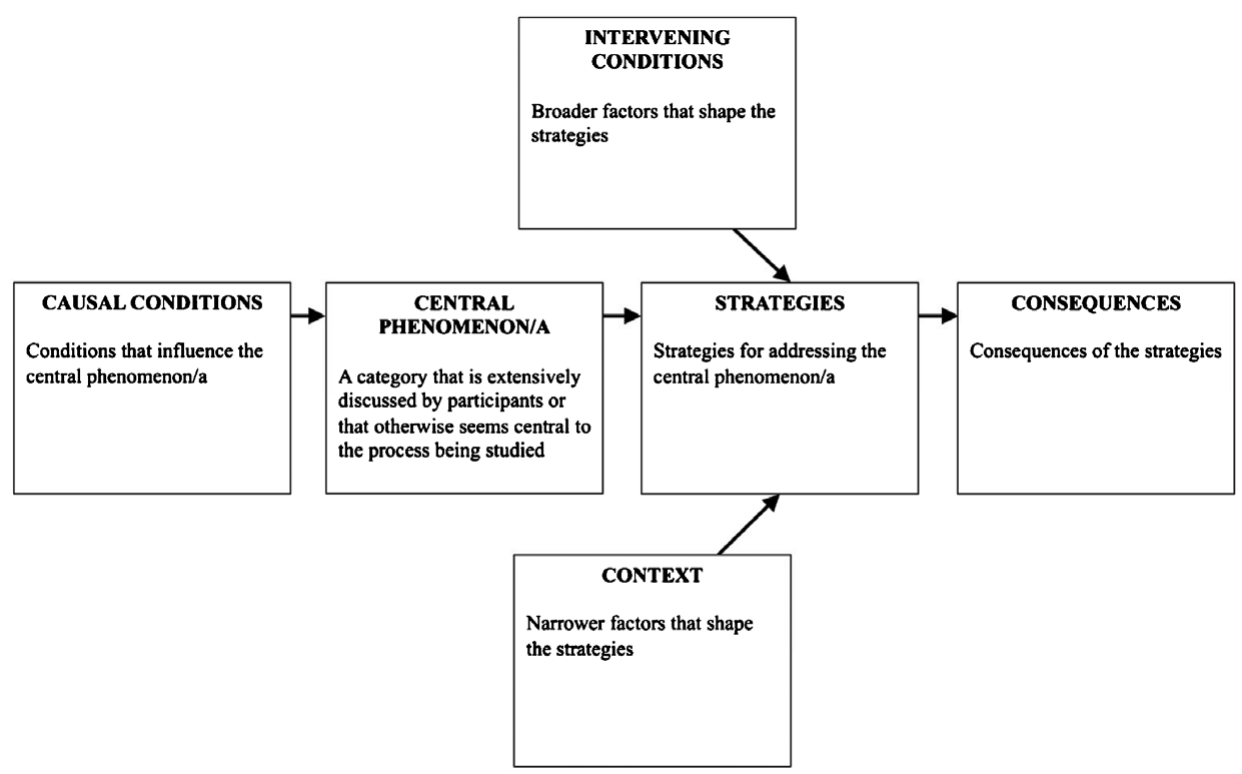

Figure 1. Straussian grounded theory framework.

It is important to note that the boxes represent coding categories rather than constructs that we typically associate with this sort of figure, and the names of the coding categories-for example, causal conditions, consequences-do not imply causal relationships in an experimental sense. Adapted from Qualitative Inquiry and Research Design: Choosing Among Five Approaches (pp. 160-161, 293), by J. W. Creswell, 2007, Thousand Oaks, CA: Sage. Copyright 2007 by Sage. See also "Constructions of Survival and Coping by Women Who Have Survived Childhood Sexual Abuse," by S. L. Morrow \& M. L. Smith, 1995, Journal of Counseling Psychology, 42, p. 27. Copyright 1995 by the American Psychological Association.

"jumping off" point for training I-O psychologists in qualitative research approaches.

\section{It's Like Doing a Job Analysis}

We show parallels between job analysis and grounded theory by presenting them in steps. However, the steps in these processes are usually not linearly or discretely conducted. Grounded theory specifically highlights the "constant comparative" method of doing data analysis while data are being collected. The same is generally true of job analysis: The job analyst develops ideas of the important tasks or skills as while reading materials related to the job, and this idea may shift as more materials are digested, interviews are conducted, observations are made, the report is drafted, and so on. For both of these processes, the job analyst/grounded theorist will bounce between steps before arriving at the final job analysis report or grounded theory. 
Job and work analysis are routinely noted as the foundation of I-O psychology as it drives all other employment decisions (e.g., hiring, training, performance management), but job analysis merits attention in its own right (Morgeson \& Dierdorff, 2011). Like job analysis, qualitative methods should be considered foundational to our field, providing a grounded understanding of what we are researching and driving future research. However, through comparison with job analysis, we hope to illustrate for I-O psychologists the fact that qualitative methods are empirical research methods on their own and should not be considered merely "pre-science" (Pratt \& Bonaccio, p. 704) done only in order to get down to the real science of having respondents circle a number on a survey page.

\section{Before You Start: The Purpose Drives Data Collection}

In both job analysis and grounded theory, there is some specific purpose driving and directing the process. In job analysis, perhaps the organization is interested in training-which would direct the job analyst to focus on trainable knowledge, skills, and abilities (KSAs) or KSAs that distinguish new and seasoned employees - or perhaps the organization is interested in hiringwhich would direct the job analyst to focus on KSAs that are appropriate for and amenable to selection tool development. In grounded theory, the researcher is interested in some phenomenon or process-and more specifically, the theory underlying it-so data collection is designed to be congruent with this purpose (Creswell, 2007).

\section{"All Is Data"}

In job analysis, a common preliminary step is to dig up and digest as much material about the job as possible-such as employee handbooks, the company website, past job ads, manuals for machinery used on the job, organizational charts, current news about the company, and so on-prior to any further data collection (Morgeson \& Dierdorff, 2011). This provides the researcher with a depth of understanding about the job and the company. Similar to the approach of digesting all available information, the Glaserian tradition of grounded theory asserts that "all is data" (Glaser, 2001, p. 145). Everything that the researcher encounters-whether it is qualitative data, quantitative data, directly related to the project or not, actual content or the delivery style, and so on-is used in analysis. In the Straussian tradition, interview and other qualitative data are typically collected, which also provide the researcher with a rich understanding of the topic of interest. Processing large amounts of information to develop a broad understanding is one common skill across job analysis and grounded theory. 


\section{To the Field!}

Many similar data collection techniques are typically used in both job analysis and grounded theory, which means that the skills developed in one realm can likely easily transfer to the other. Often in both techniques, job analysts/grounded theorists observe and conduct interviews with appropriate parties-namely, subject matter experts (SMEs) in job analysis parallel the informants in ground theory (see focal article)-either in groups or individually. Many of the same challenges will likely be encountered with data collection in both realms, including identifying and contacting SMEs, conducting and recording observations, facilitating and recording interviews, being comfortable with semistructured protocols for data collection, and adapting data collection based on concurrent data analysis. These skills can be difficult to learn, but many of the basic skills learned by doing job analysis can be transferred to doing grounded theory and vice versa.

So when is the analyst/theorist done collecting data in the field? Both processes emphasize developing a comprehensive understanding while data are being collected, the quality of which is determined based on the data itself. Grounded theory emphasizes data collection until the point of theoretical saturation, when no new information is being collected. However, saturation can't be determined unless the theorist is analyzing data while they are being collected. Therefore, while collecting data, the theorist must simultaneously begin to develop the theory and compare it with new data in order to determine when data collection is providing diminishing returns for the theory. Likewise, the job analyst must begin to develop the job description and determine its required tasks and KSAs in order to determine whether there's a comprehensive understanding of the job. If gaps are identified in understanding some component of the job-much like in grounded theorythen the job analyst should return to the field, perhaps doing a follow-up interview with an SME. In both cases, the process is iterative, and the end of data collection depends on data analysis.

\section{Induce/Deduce}

The process of developing tasks and KSAs or grounded theory codes and categories is inductive-built up from the data. During this process, the job analyst/grounded theorist develops the hard-earned skill of boiling down rich, qualitative data into a more concise version of the findings. Working through rich data to an accurate but succinct list of KSAs and job descriptions will transfer to the similar process done with grounded theory, in which the theorist identifies codes, concepts, and categories to complete the theory. 
However, again, we emphasize that this qualitative research approach is iterative-after inducing a theory, the grounded theorist should also ensure that the theory deductively fits the data and adjust the theory as needed. Therefore, grounded theory is not purely inductive (see focal article) but rather combines induction with some deduction through the continuous verification that the emerging theory fits the data. We suggest that the same is true of a thorough job analysis: The analyst often develops a list of tasks and KSAs but should revisit the data repeatedly to ensure that the list is an accurate representation of the richer data collected on the job and organization.

\section{Reporting}

Although job analysis often includes quantitative data collection (discussed in the following section) following the development of a list of tasks and KSAs, grounded theory typically uses mainly qualitative data and qualitative analyses (Creswell, 2007). Therefore, we focus here on a final common requirement of job analysis and grounded theory: the need to report rich, qualitative data. In both techniques, the final product often is a detailed narrative summary. In job analysis, analysts generally develop a job description and a job analysis report. This product provides the job analyst with practice not only in collecting and analyzing qualitative data but also in producing a qualitative product. Likewise, in grounded theory, the theorist must "tell the story" of the theory, a task that can be especially challenging for new grounded theory researchers (Corley, 2012, p. 511). However, I-O psychologists have already practiced this skill by producing job descriptions and job analysis reports.

\section{Though They're Not Exactly the Same}

Of course, job analysis and grounded theory are not exactly the same. The core difference is the purpose and corresponding frameworks of each analysis. Grounded theory is used to understand a process or phenomenon, and researchers can thus use a framework of identifying the central concept underlying the process or phenomenon, what precedes it, what results from it, and what factors shape what results from it (Creswell, 2007). Although job analysis can be conducted for a variety of purposes (e.g., hiring versus training), at its core, job analysis is used to understand a job in a particular context. Like in qualitative methods (see focal article), job analysis emphasizes understanding the context in which the job occurs. However, the framework for job analysis differs from that of grounded theory: Rather than identifying one core concept underlying the job and factors affecting it, job analysis approaches generally emphasize understanding all important tasks done on the job, the skills or competencies needed to do the job, and the context in 
which the work is done. For both analyses, there are "buckets" to fill, but their buckets differ.

A second difference that's perhaps the largest between these two analyses is that grounded theory is a stand-alone qualitative research approach. The data are qualitative and so is the analysis of that data-the researcher's units of analysis and reporting are concepts and ideas. By contrast, many approaches to job analysis incorporate some variation of quantitative data collection, analysis, and reporting, such as gathering and reporting averages of SMEs' ratings of the frequency of tasks or the importance of KSAs. However, this means that experience with job analysis also lends itself to developing qualitative researchers who can address the same question in greater depth by applying multiple research approaches to a single issue. For example, we could expect that a job analyst turned qualitative researcher would be able to develop and conduct mixed methods or quantitative follow-up studies or design qualitative studies that extend beyond what can be examined in a strictly quantitative study.

Of course, as in job analysis, there are many different approaches within qualitative research; grounded theory is just one of these approaches. Upon one's first encounter, the world of qualitative research approaches and procedures can seem vast and intimidating. However, when we see the parallels between those varied approaches and the many approaches to job analysis, we can more comfortably venture into the world of qualitative research. Although grounded theory provides a helpful framework for illustrating the similarities with job analysis, learning other qualitative approaches-such as ethnography or case studies-will also likely be facilitated by the skills that I-O psychologists develop through job analysis.

\section{Conclusion}

Our take-home message here is that fear (or aversion or disdain) of the "unknown" regarding qualitative research is not necessary for I-O psychologists. We know how to gather, analyze, and report rich, qualitative data and have been doing so since the birth of our field. Though the purpose, content, and other details will change as we move toward using qualitative research approaches, we are confident that I-O psychologists have applicable, transferable skills from learning and doing job analysis.

\section{References}

Corley, K. (2012). Publishing in AMJ_Part 7: What's different about qualitative research? Academy of Management Journal, 55, 509-513. doi:10.5465/amj.2012.4003

Creswell, J. W. (2007). Qualitative inquiry and research design: Choosing among five approaches. Thousand Oaks, CA: Sage.

Glaser, B. (2001). The grounded theory perspective: Conceptualization contrasted with description. Mill Valley, CA: Sociology Press. 
Glaser, B., \& Strauss, A. (1967). The discovery of grounded theory. London, UK: Weidenfeld and Nicholson.

Morgeson, F. P., \& Dierdorff, E. C. (2011). Work analysis: From technique to theory. In S. Zedeck (Ed.), APA handbook of industrial and organizational psychology (Vol. 2, pp. 3-41). Washington, DC: APA.

Morrow, S. L., \& Smith, M. L. (1995). Constructions of survival and coping by women who have survived childhood sexual abuse. Journal of Counseling Psychology, 42, 24-33. doi:10.1037/00220167.42.1.24

Pratt, M. G., \& Bonaccio, S. (2016). Qualitative research in I-O psychology: Maps, myths, and moving forward. Industrial and Organizational Psychology: Perspectives on Science and Practice, 9(4), 693-715.

Society for Industrial and Organizational Psychology. (2016). Guidelines for education and training in industrial-organizational psychology. Bowling Green, $\mathrm{OH}$ : Author. Retrieved from http://www. siop.org/Instruct/SIOP_ET_Guidelines_2016.pdf 